\title{
The Outcome of Medical Management of Cornual Ectopic Pregnancy: Two Case Reports
}

\author{
Papa Dasari, Sharada Patra' \\ Dept of Obs \& Gynae, JIPMER, Puducherry
}

\begin{abstract}
Cornual pregnancy is a very rare and dangerous type of ectopic pregnancy associated with high mortality. Preserving fertility is an important issue especially when it occurs in nulliparous women and hence conservative treatment with medical therapy is the first option.

Two cases of early first trimester Interstitial pregnancy diagnosed by USG in Nulliparous women were managed conservatively by medical therapy, Multidose methotrexate and Mifepristone combination was employed. One of them responded by expulsion of the products even though she had very high $\beta$ HCG levels. The other though responded initially had increased echogenecity and size of the mass. Laparotomy was undertaken for symptoms of rupture showed only threatened rupture of the cornual pregnancy and haemoperitoneum due to corpus luteal cyst rupture. Conservative surgery i.e., cornual resection could be performed successfully without much blood loss.
\end{abstract}

Comment: Multidose methotrexate followed by Mifepristone is a safe option in medical management of ectopic pregnancy and the patient should be followed up closely.

Key words : Cornual ectopic pregnancy, Mifepristone, Methotrexate, cornual resection

\section{Introduction}

Cornual or Interstitial ectopic pregnancy is a rare condition accounting for $1.8 \%$ of all ectopic pregnancies and $0.01 \%$ of all pregnancies ${ }^{1}$. Early diagnosis and proper management is of utmost importance to prevent mortality and also to preserve fertility. A mortality rate up to $2 \%$ is reported as the pregnancy advances to second trimester and when unrecognised, results in catastrophic haemorrhage and death. The traditional treatment includes cornual resection/hysterectomy. We describe here the outcome of two cases of cornual pregnancies in which medical management was instituted.

Case 1: A 31 year old fourth gravid with previous three first trimester abortions complained of two months amenorrhea and spotting for one day. There was no history of pain abdomen. On examination, she was anxious with a pulse rate of $96 / \mathrm{min}$ and had no pallor. She was normotensive and other systemic examination was normal. On per speculum examination, cervix and vagina were healthy and there was no bleeding. On per vaginal examination, uterus was soft and was 10 weeks

Correspondence

Dr.Papa Dasari. MD, DGO

Prof, Dept of Obs \& Gynae,

JIPMER, Puducherry, India size and anteverted. Transvaginal USG revealed an irregular sac at right cornual region measuring $16 \mathrm{X} 14 \mathrm{~mm}$ without any fetal pole. Decidual thickness was $20 \mathrm{~mm}$. Serum $\beta$ HCG was $21,530 \mathrm{mIU} / \mathrm{ml}$ on 19/3/08 2days after admission. She was given multidose methotrexate 1 $\mathrm{mg} / \mathrm{kg}$ body weight (from 19/3/08) on day1,3,5,7,9 alternating with injection folinic acid. USG done on $27 /$ $3 / 08$ showed the presence of crumpled cornual gestational sac (Fig 1) and repeat Serum $\beta$ HCG on the

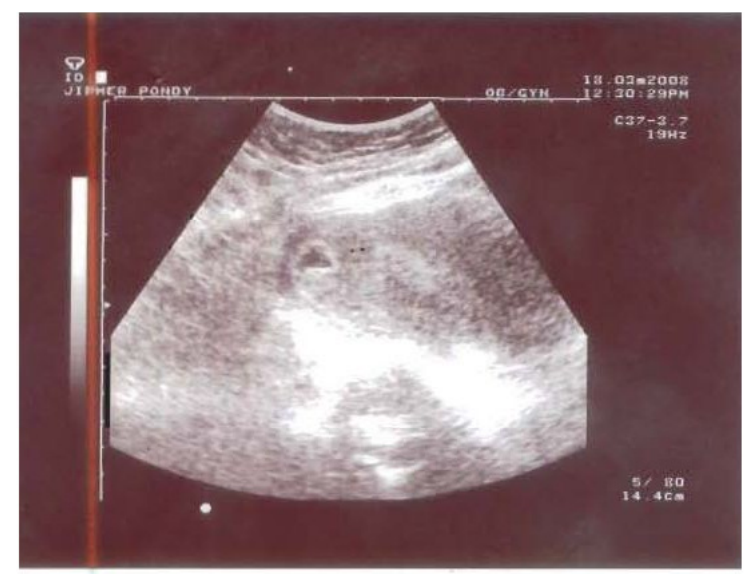

Fig 1. USG showing cornual ectopic pregnancy 


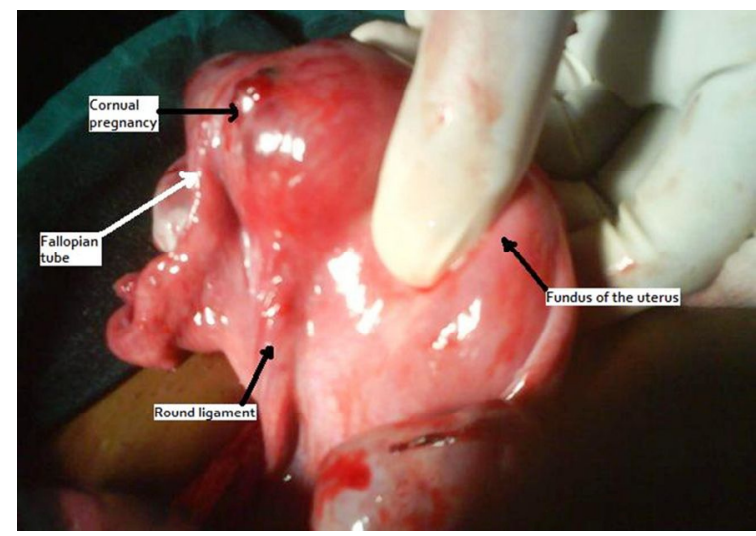

Fig 2. Cornual ectopic pregnancy

same day was $21,356 \mathrm{mIU} / \mathrm{ml}$. She was given tablet Mifepristone $400 \mathrm{mg}$ orally on 28/3/08 and she expelled products on 30/3/08. USG showed empty cavity. HPE of products was reported as gestational hyperplastic endometrium and decidua.

Case 2: A 22 year primigravida was admitted as a case of threatened abortion at 9 weeks of pregnancy. USG evaluation showed gestational sac of $29 \mathrm{~mm}$ X $27 \mathrm{~mm}$ in right cornual region with a small fetal pole without any cardiac activity. She was given tablet Mifepristone $200 \mathrm{mg}$ orally and single dose methotrexate which had no effect. Then she was given multidose methotrexate therapy with folinic acid. $\beta$ HCG which was $3,721 \mathrm{mIU} /$ $\mathrm{ml}$ has reduced to $892.60 \mathrm{~m} \mathrm{IU} / \mathrm{ml}$ after 2 weeks and the sac size measured 1.5 to $1.6 \mathrm{~cm}$. $\beta$ HCG reduced further to $169 \mathrm{~m} \mathrm{IU} / \mathrm{ml}$ after another week and the patient did not have any clinical symptoms of pain or fainting attacks. $\beta$ HCG was $125 \mathrm{mIU} / \mathrm{ml}$ measured during the following week. USG done after another week i.e 3 weeks after administration of multidose methotrexate showed echogenic mass in the place of the gestational sac measuring $3.7 \mathrm{X} 4 \mathrm{~cm}$. There was no free fluid in POD. She was evaluated again on the next day. On per vaginal examination, cervix movement excitation was present and uterus felt normal with a tender adnexal mass and repeat USG showed $4 \times 4 \mathrm{~cm}$ heteroechoeic mass at the right cornual region along with free fluid in POD. Emergency laparotomy was undertaken which revealed right cornual ectopic with impending rupture but there was no bleeding from this site. Corpus luteal cyst was on the left side and there was bleeding from this because of its rupture. Right cornual resection and right partial salphigectomy was done. Left sided ruptured corpus luteal cyst was sutured. The postoperative period was uneventful. Histopathological examination showed villi and membranous decidua along with blood vessels and myometrium.

\section{Comment}

Preserving fertility is the dire necessity in both the cases described above. Medical management is the first line of option in such cases. Medical treatment is reported to be associated with high failure rates and surgical treatment involving cornual resection has decreased fertility rate and increased uterine rupture in subsequent pregnancies ${ }^{2}$. A combined approach is also has been advocated ${ }^{3}$.

Of the conservative management options for cornual ectopic pregnancies, two cases where expectant management was given was described by Ilan E. Timortritsch and collegues. At the time of diagnosis these pregnancies were non viable and had very low serum HCG levels. But these women needed follow up for long period of time and $\beta$ HCG came down to normal levels only after a period of 105 days and 70 days in the two cases. Local injection of Kcl and methotrxate in to the gestational sac in viable cornual pregnancies resulted in early resolution of cornual ectopic as evidenced by the return of $\beta$ HCG 42 and 35 days respectively in two other cases ${ }^{4}$. However failure of local injection of methotrexate in to the gestational sac was reported in two cases, one of them resulting in rupture and the other responding to addition of intravenous high dose methotrexaate infusion ${ }^{1}$. In our first case expectant management could not be considered because of high $\beta$ HCG levels though she had a small gestational sac and showed features of non-viable pregnancy. We have given multidose methotrexate which did not result in significant decrease of $\beta$ HCG immediately after the course and hence we treated with Mifepristone, an antiprogesterone after which she expelled the sac in $48 \mathrm{hrs}$ and we consider this may be due to the combined effect of both the drugs. In the second case, because of the experience with first case, we tried combination of Mifepristone and single dose intramuscular methotrexate. However this did not result in the expected response, hence multidose methotrexate therapy was instituted. Even though $\beta$ HCG levels have dropped after multidose methotrexate, she showed increase in the size and echogenicity in the form of mass in place of gestational sac most probably due to separation of chorionic plate resulting in haemorrhage following Mifepristone administration. The initial intramuscular dose of methotrexate may not have been adequate to result in the death of the trophoblastic tissue. Hence it may be inferred that multi dose methotrexate may be given first to bring about death of the trophoblast more efficiently than Mifepristone.

In a review of large series, Fisch and his collegues concluded methotrexate to be a safe option for conservative treatment of cornual ectopics ${ }^{5}$. Mefipristone (600mg) and methotrexate $950 \mathrm{mg} / \mathrm{m}^{2}$ ) combination resulted in a faster resolution of unruptured tubal ectopic pregnancies when compared to methotrexate alone in a RCT which included 50 
women. The effect of combined treatment is also more pronounced when $\beta$ HCG levels are high ${ }^{6}$. There is no consensus in the dose or number of methotrexate injections to be used in treating interstitial pregnancies successfully. The failure with methotrexate therapy has been attributed to the increased vascularity of interstitial pregnancies because of their location and it has been suggested that multidose methotrexate should be used when the $\beta$ HCG levels are $>1000 \mathrm{mIU} / \mathrm{ml}^{7}$. Persistence of cornual pregnancy after failure of multidose methotrexate can be managed by hysteroscopic evacuation under Ultrasound guidance. ${ }^{8}$

We managed this failure again by medical method; however in the second case haemoperitoneum was due to corpus luteal cyst rupture. Though there were signs of impending rupture of cornual pregnancy in this case, there was not much of bleeding at the time resection.

\section{References}

1. Moir D, McMohan K. Cornual ectopics- two case studies. ASUM Ultrasound bulletin 2004;7(4):2325 .

2. Ross R, Leindheim SR, Olive DL, Pritts EA. Cornual gestation: a systemic literature review and two case reports of novel treatment. J Minim
Invasive Gynecol. 2006;13 (1): 74-78.

3. Shamma AI Inizi, Masroor Cheema,Vincent B. Conservative laparoscopic and medical treatment for Cornual pregnancy. The internetJournal of Gynecology and Obstetrics 2007;7: 1.(www.ispub.com)

4. Timor-Tritsch E I, Monteagudo A, Matera C, Veit R C. Sonographic evolution of cornual pregnancies treated without surgery. Obstet Gynecol.1992;79:1044-9.

5. Fisch JD, Oritz B, Tazuke S, Chitkara U, Giudice L. Medical management of interstitial ectopic pregnancy- a case report and literature review. Hum Reprod. 1998; 13 (7): 1981-1986.

6. Gazvani M R, Baruah D N, Alfirevic Z, Emery S J.Mifepristone in combination with methotrexate for the medical treatment of Tubal pregnancy: a randamised controlled trial. Hum Reprod, 1998;13(7):1987-1990.

7. Barnhart K, Spandorfer S, Coutifaris C. Medical treatment of Interstitial pregnancy - a report of three unsuccessful cases. J Reprod Med. 1997; 42: 521-524.

8. Sanz Luis E,Verosko J. Hysteroscopic management of cornual ectopic pregnancies. Obstet and Gynecol. 2002;99 (5):941-944. 